\title{
The problem solution of deformable power surfaces from anisotropic material interaction in elements of structure constructions
}

\author{
Boris Ivanov ${ }^{1}$, Alexander Pischulev ${ }^{1, *}$, and Galina Reazanova ${ }^{1}$ \\ ${ }^{1}$ Samara State Technical University, Academy of Architecture and Civil Engineering, \\ Molodogvardeyskaya St., 194, Samara, 443001, Russia
}

\begin{abstract}
The paper presents an analytical method of examining deformable power surfaces from anisotropic material and their interaction. Materials with anisotropic properties are often used in the elements of transport structures as well as in building construction [1-7]. Most frequently the following materials are used in construction: rolled metal products, products obtained by drawing, wires of various kinds, reinforced concrete structures, bridge bearings, supporting walls and other structures, composite materials, plastics and other [8-13]. For forecasting strength properties of structures strength joints and their behaviour under various external influences, the authors developed a method of calculation of their stress-strain behaviour [9]. This method can be applied to strength joints of complex form made of anisotropic elastic elements under specific conditions of deformation of the contact surfaces.
\end{abstract}

\section{Introduction}

The problems of searching for a strained deformed state of a solid body under variable kinematic and static boundary conditions are related to problems of parametric optimization or synthesis. Such problems can be formulated in a more traditional form, that is, in the form of searching for an optimal form of construction under varying boundary conditions [14-32]. Construction optimization can be organized only for its individual elements [1426], separately for its joint connections [27-32] or solved in a complex for joints and elements. The problem solution can be obtained by analytical methods [9] or numerical ones [14-32].

\section{Materials and methods}

The problem solution is shown by the analytical method. In the presented problem, the following interaction conditions of the contact surfaces are considered:

1. Movements are specified on the entire surface of the deformable solid body. The area in which they are different from zero is only a certain time-varying part of the surface of the body.

\footnotetext{
* Corresponding author: pishulev@yandex.ru
} 
2. External forces are set on the entire surface of the deformable element. The area in which these efforts are different from zero is only a certain time-varying part of the surface of the body.

3. Parts of the body with given movements and given forces are different parts of the surface of the deformed body.

\section{Equations and mathematics}

The deformable body is assumed to have finite dimensions, its material being homogeneous. Its state is described by the following problem in the theory of elasticity:

$$
\left.\begin{array}{l}
\sigma_{i j, j}(\mathbf{x}, t)=F(\mathbf{x}, t) ; \varepsilon_{i j}(\mathbf{x}, t)=\frac{1}{2}\left(u_{i, j}(\mathbf{x}, t)+u_{j, i}(\mathbf{x}, t)\right) \\
\sigma_{i j}(\mathbf{x}, t)=\Gamma_{i j p q} \cdot \varepsilon_{p q}(\mathbf{x}, t) ;\left.u_{i}(\mathbf{x}, t)\right|_{s(t)}=u_{i 0}(\mathbf{x}, t)
\end{array}\right\}
$$

Let's represent the Green's function $G_{m n}(\mathbf{x}, \mathbf{y}, t)$ boundary-value problem (24) in the form of a sum of two functions:

$$
G_{m n}(\mathbf{x}, \mathbf{y}, t)=K_{m n}(\mathbf{x}, \mathbf{y}, t)+W_{m n}(\mathbf{x}, \mathbf{y}, t)
$$

where $K_{m n}(\mathbf{x}, \mathbf{y}, t)$ - the fundamental solution of the Lame operator is the KelvinSomigliana tensor, a $W_{m n}(\mathbf{x}, \mathbf{y}, t)$ - boundary-value problem solution:

$$
\left.\begin{array}{l}
(L)_{e m} W_{m n}(\mathbf{x}, \mathbf{y}, t)=0 \\
\left.W_{m n}(\mathbf{x}, \mathbf{y}, t)\right|_{s(t)}=-K_{m n}\left(\mathbf{x}_{s,} \mathbf{y}, t\right)
\end{array}\right\}
$$

Boundary-value problem solution (1) can be presents as:

$$
u_{i}(\mathbf{x}, t)=\int_{v} G_{i j}(\mathbf{x}, \mathbf{y}, t) F_{j}(\mathbf{y}, t) d \mathbf{y}-\int_{s} u_{k 0}(\mathbf{x}, t) \overline{\sigma_{i l m}}\left(G_{m j}(\mathbf{x}, \mathbf{y}, t)\right) n_{j}(\mathbf{x}, \mathbf{y}, t) d S
$$

To determine the values of a function $W_{m n}(\mathbf{x}, \mathbf{y}, t)$ on body surface:

$$
W_{m n}\left(\mathbf{x}_{s}-\mathbf{y}, t\right)=\frac{1}{(2 \pi)^{3}} \int_{R^{3}}\left((L)_{m n}{ }^{*}(\mathbf{k})\right)^{-1} \cdot e^{i \mathbf{k}\left(\mathbf{x}_{s}-\mathbf{y}\right)} d \mathbf{k}
$$

Problem solution (3) is defined as the volume potential

$$
W_{m n}(\mathbf{x}, t)=\int_{V_{1}} K_{m p}(\mathbf{x}-\mathbf{y}, t) F_{p n}(\mathbf{y}, t) d \mathbf{y}
$$

The volume of integration $V_{l}$ contains volume $V$ occupied by the deformed body, and the tensor $F_{p n}(\mathrm{y}, t)$ is mass forces tensor distributed in the volume $V 1$.

The integration in (6) in fact was carried out from the difference in the volumes $V$ and $V 1$. The function $W_{n m}(\mathrm{x}, t)$ represented by the formula (6) satisfies the equation of the boundary value problem (1) by the properties of the fundamental solution, and the selection of $F_{p n}(\mathrm{y}, t)$ is carried out taking into account the boundary conditions of problem (3).

Carried out a number of transformations, using the equalities to define the tensor components $N(\mathrm{x} s) e^{-i k \times s}$, integrating the intermediate expression on the surface $S$ with the 
subsequent transition to integration on the surface $V$ and introducing a number of notations with the expansion of the divergence operator, taking into account the convolution theorem, made it possible to determine the Fourier images of the coordinates of the mass force:

$$
\left.\begin{array}{c}
W_{m q}^{*}(\mathbf{k}, t)=\delta_{m l}\left\{-i k_{l} K_{l p}^{*}(\mathbf{k}, t)+K_{l p}^{*}(\mathbf{k})\right\} F_{p q}^{*}(\mathbf{k}, t) \\
K_{l p}^{*}(\mathbf{k}, t)=\int_{V_{z}} e^{-i k \mathbf{z}} \cdot K_{l p}(\mathbf{z}, t) d \mathbf{z} ; \\
K_{l p, l}^{*}(\mathbf{k}, t)=\int_{V_{z}} e^{-i k \mathbf{z}} \cdot \frac{\partial}{\partial x_{l}} K_{l p}(\mathbf{z}, t) d \mathbf{z}
\end{array}\right\}
$$

In formulae (8) integrating volumesя $V_{2}$ are defined by $V_{1}, V$ and the correlation $\mathbf{z}=\mathbf{x}-\mathbf{y}$. In the congruence (7) equation $\delta_{m l}\left\{-i k_{l} K_{l p}^{*}(\mathbf{k}, t)+K_{l p}^{*}(\mathbf{k}, t)\right\}$ is a matrix of the third order, the elements of which are functions of the parameter $\mathbf{k}$, and the determinant is different from zero. From the construction of the matrix $M_{i n}(\mathbf{k}, t)$ inverse to the matrix of the system, we obtain the formula

$$
f_{i j}(\mathbf{k}, t)=M_{i n}(\mathbf{k}, t) \cdot W_{n j}^{*}(\mathbf{k}, t)
$$

Allowing to write down $W_{m n}(\mathbf{x}, t)$ - the solution of the problem (3) in the form:

$$
W_{m n}(\mathbf{x}, t)=\int_{V_{1}} K_{m p}(\mathbf{x}-\mathbf{y}, t)\left\{\frac{1}{(2 \pi)^{3}} \int_{R^{3}} e^{i R y} M_{p l}(\mathbf{k}, t) W_{\mathrm{ln}}^{*}(\mathbf{k}, t) d \mathbf{k}\right\} d \mathbf{y}
$$

From (2) и (10) the Green's function $G_{m n}(\mathbf{x}-\mathbf{y}, t)$ boundary problem (1) we get:

$$
\begin{aligned}
& G_{m n}(\mathbf{x}-\mathbf{y}, t)=K_{m n}(\mathbf{x}-\mathbf{y}, t)+\int_{V_{1}} K_{m p}\left((\mathbf{x}-\mathbf{y}, t)-\mathbf{y}_{1}\right) \times \\
& \times\left\{\frac{1}{(2 \pi)^{3}} \int_{R^{3}} e^{-i k \mathbf{y}_{1}} \cdot M_{p l}(\mathbf{k}, t) W_{\mathrm{ln}}^{*}(\mathbf{k}, t) d \mathbf{k}\right\} d \mathbf{y}
\end{aligned}
$$

From the proof of the theorem it follows that the tensor function $W_{m n}(\mathbf{x}, t)$, defined by (10) is a solution of the boundary value problem (3) and relation (11) determines the Green's function of the boundary value problem (1).

External forces are prescribed on the entire surface of the deformable element, and the area in which these forces are different from zero also constitute just a part of the body surface, and vary some time later.

In this case, the stress-strain state of the body is described by the following boundaryvalue problem of the theory of elasticity:

$$
\left.\begin{array}{l}
\sigma_{i j, j}(\mathbf{x}, t)=F_{i}(\mathbf{x}, t) ; \varepsilon_{i j}(\mathbf{x}, t)=\frac{1}{2}\left\{u_{i, j}(\mathbf{x}, t)+u_{j, i}(\mathbf{x}, t)\right\} \\
\sigma_{i j}(\mathbf{x}, t)=\Gamma_{i j p q} \cdot \varepsilon_{p q}(\mathbf{x}, t) ;\left.\sigma_{i j}(\mathbf{x}, t) n_{j}(\mathbf{x}, t)\right|_{S(t)}=P_{i}\left(\mathbf{x}_{s}, t\right)
\end{array}\right\}
$$

The Green's function $G_{m n}(\mathbf{x}, \mathbf{y}, t)$ of boundary problem (1) as in the previous case, is representable as a sum: 


$$
G_{m n}(\mathbf{x}, \mathbf{y}, t)=K_{m n}(\mathbf{x}, \mathbf{y}, t)+W_{m n}(\mathbf{x}, \mathbf{y}, t)
$$

$W_{m n}(\mathbf{x}, \mathbf{y}, t)-$ is a solution of the boundary value problem:

$$
\left.\begin{array}{l}
(L)_{l m} W_{m n}(\mathbf{x}, \mathbf{y}, t)=0 \\
\left.\sigma_{i j}(\mathbf{x}, \mathbf{y}, t) n_{j}(\mathbf{x})\right|_{S(t)}=-\left.\sigma_{i j}(K) n_{j}(\mathbf{x})\right|_{S(t)}=P_{i}^{*}\left(\mathbf{x}_{S}, \mathbf{y}, t\right)
\end{array}\right\}
$$

After the introduction of $G_{m n}(\mathbf{x}, \mathbf{y}, t)$ boundary problem solution (12) present in the form:

$$
u_{i}(\mathbf{x}, t)=\int_{V} G_{i j}(\mathbf{x}, \mathbf{y}, t) F_{j}(\mathbf{y}, t) d \mathbf{y}-\int_{S} G_{i j}(\mathbf{x}, \mathbf{y}, t) P_{j}\left(\mathbf{y}_{S}, t\right) d S
$$

Surface value function:

$$
\left.\sigma_{i j}\left(\mathbf{x}_{S}, \mathbf{y}, t\right) n_{j}\left(\mathbf{x}_{S}\right)=-\frac{1}{(2 \pi)^{3}} \sigma_{i j}\left(\int_{R^{3}} K^{*}(\mathbf{k}, t)\right)^{-1} \cdot e^{i k x_{S}} d \mathbf{k}\right) n_{j}\left(\mathbf{x}_{S}\right)=P_{i}^{W}\left(\mathbf{x}_{S}, \mathbf{y}, t\right)
$$

Problem solution (14) is in the form:

$$
W_{m n}(\mathbf{x}, t)=\int_{V_{1}} K_{m p}(\mathbf{x}-\mathbf{y}, t) F_{p n}(\mathbf{y}, t) d \mathbf{y}
$$

The choice $F_{p n}(\mathbf{y}, t)$ is carried out from the condition for the satisfaction of boundary conditions (12).

The Fourier transform of the boundary problem (14), integration by parts, the application of the Ostrogradskii-Gauss formula and the convolution theorem for the Fourier images of the stress tensor coordinates made it possible to obtain:

$$
\begin{aligned}
& \sigma_{\alpha \beta \gamma}^{*}(\mathbf{k}, t)=K_{\alpha \beta \gamma}^{\sigma}(\mathbf{k}, t) F_{\delta \gamma}^{*}(\mathbf{k}, t) \\
& \sigma_{\alpha \beta \gamma}^{*}(\mathbf{k}, t)=\int_{V} e^{i k \cdot x} \cdot \sigma_{\alpha \beta \gamma}(\mathbf{x}, t) d \mathbf{x} \\
& K_{\alpha \beta \gamma}^{\sigma}(\mathbf{k}, t)=\int_{V_{K}} e^{i k \cdot \mathbf{x}} \cdot \sigma(K)_{\alpha \beta \delta}(\mathbf{x}, t) d \mathbf{x} \\
& F_{\delta \gamma}^{*}(\mathbf{k}, t)=\int_{V_{1}} e^{i k \cdot \mathbf{x}} \cdot F_{\delta \gamma}(\mathbf{x}, t) d \mathbf{x} \\
& P_{\alpha \gamma}^{* W}(\mathbf{k}, t)=i \mathbf{k}_{\beta} K_{\alpha \beta \delta}^{\sigma}(\mathbf{k}, t) F_{\delta \gamma}^{*}(\mathbf{k}, t)
\end{aligned}
$$

The system of linear algebraic equations (20), equivalent to the original boundary-value problem, has also a unique solution because of the uniqueness of the solution, and, consequently, its determinant is different from zero. $i \mathbf{k}_{\beta} K_{\alpha \beta \delta}^{\sigma}(\mathbf{k}, t)$ has a reverse matrix $K_{\alpha \delta}(\mathbf{k}, t)$, using which, the solution of the system of equations (20) can be presented as:

$$
F_{\delta \gamma}^{*}(\mathbf{k}, t)=K_{\delta \alpha}(\mathbf{k}, t) P_{\alpha \gamma}^{* W}(\mathbf{k}, t)
$$


From (21) using the inverse Fourier transform for $W_{m n}(\mathbf{x}, t)$ a relation is got:

$$
W_{m n}(\mathbf{x}, t)=\int_{V_{1}} K_{m p}(\mathbf{x}-\mathbf{y}, t)\left\{\frac{1}{(2 \pi)^{3}} \int_{R^{3}} e^{i k \cdot y} \cdot K_{p q}(\mathbf{k}, t) \cdot P_{q n}^{* W}(\mathbf{k}, t) d K\right\} d \mathbf{y}
$$

From the proofs of the theorems presented in the paper:

- function $W_{m n}(\mathbf{x}, t)$, defined by relation (22), satisfies the system of equations of the boundary-value problem (14);

- function $W_{m n}(\mathbf{x}, t)$, defined by relation (22), satisfies boundary problem conditions (14) and (22) boundary problem solution (14);

According to the statement of the problem - external forces are specified on the part surface of the deformable element, and movements on the parts. The areas in which they are given make up just a part of the body surface and they change some time later.

This loading type is described by the following problem in the theory of elasticity:

$$
\left.\begin{array}{l}
\sigma_{i j, j}^{*}(\mathbf{x}, t)=F_{i}^{*}(\mathbf{x}, t) ; \varepsilon_{i j}^{*}(\mathbf{x}, t)=\frac{1}{2}\left\{u_{i, j}^{*}(\mathbf{x}, t)+u_{j, i}^{*}(\mathbf{x}, t)\right\} \\
\sigma_{i j}^{*}(\mathbf{x}, t)=\Gamma_{i j p q} \cdot \varepsilon_{p q}^{*}(\mathbf{x}, t) ; \\
\left.u_{i}^{*}(\mathbf{x}, t)\right|_{S_{u(t)}}=u_{i o}^{*}\left(\mathbf{x}_{s}, t\right) ;\left.\sigma_{i j}^{*}(\mathbf{x}, t) n_{j}(\mathbf{x})\right|_{S_{\sigma(t)}}=P_{i}^{*}\left(\mathbf{x}_{S}, t\right)
\end{array}\right\}
$$

on $S_{U}(t)$ part of the deformed body surface $U^{*}{ }_{i o}\left(\mathrm{x}_{s} t\right)$ interchanges are given, on the other part $S_{\sigma(t)}$-there are surface power $P_{i}^{*}\left(\mathrm{x}_{s}, t\right)$.

It is assumed that the material of the deformed body is anisotropic. Then, as before, the Kelvin-Somigliana tensor for the anisotropic Lamé operator makes it possible to reduce the inhomogeneous boundary value problem (23) to a homogeneous one:

$$
\left.\begin{array}{l}
\sigma_{i j, j}(\mathbf{x}, t)=0 ; \varepsilon_{i j}(\mathbf{x}, t)=\frac{1}{2}\left\{u_{i, j}(\mathbf{x}, t)+u_{j, i}(\mathbf{x}, t)\right\} \\
\sigma_{i j}(\mathbf{x}, t)=\Gamma_{i j p q} \cdot \varepsilon_{p q}(\mathbf{x}, t) ; \\
\left.u_{i}(\mathbf{x}, t)\right|_{S_{u}(t)}=u_{i o}\left(\mathbf{x}_{s}, t\right) ;\left.\sigma_{i j}(\mathbf{x}, t) n_{j}(\mathbf{x})\right|_{S_{\sigma}(t)}=P_{i}\left(\mathbf{x}_{S}, t\right)
\end{array}\right\}
$$

To solve the problem (24) the solutions of the first and second boundary value problems presented above are used.

The boundary problem solution (24) with the Stokes formula for the Lame operator can be written in the form:

$$
u_{i}(\mathbf{x}, t)=\int_{S}\left[K_{i j}\left(\mathbf{x}-\mathbf{y}_{S}, t\right) \cdot P_{j}\left(\mathbf{y}_{S}, t\right)-(\sigma)_{i j q}\left(K\left(\mathbf{x}-\mathbf{y}_{S}, t\right)\right) \cdot u_{j}\left(\mathbf{y}_{S}, t\right) \cdot n_{q}\left(\mathbf{y}_{S}\right)\right] d S
$$

Taking into account the action of the stress operator on the Kelvin-Somigliana tensor $(\sigma)_{j i q}\left(K\left(\mathbf{x}-\mathbf{y}_{S}, t\right)\right)$, division of the body surface into two parts $S_{U}$ and $S_{\delta}$ and the Fourier transform the following is obtained:

$$
\begin{aligned}
& u_{i}^{*}(\mathbf{k}, t)=K_{i j}^{*}(\mathbf{k}, t) P_{j u}^{*}(\mathbf{k}, t)+K_{i j}^{* \delta}(\mathbf{k}, t) P_{j}^{*}(\mathbf{k}, t)-(u n)_{j q}^{*}(\mathbf{k}, t)(\sigma)_{i j q}^{* u}(\mathbf{k}, t)- \\
& -(u n)_{j q}^{* \delta}(\mathbf{k}, t)(\sigma)_{i j q}^{* \delta}(\mathbf{k}, t)
\end{aligned}
$$


Where $P^{*}{ }_{j u}(\mathbf{k}, t), P_{j}^{*}(\mathbf{k}, t)$ - Fourier-images of surface forces defined over a part of the surface where the displacements are known $u(\mathbf{x}, t)$ and surface forces respectfully; $(u n)_{j q}^{*}(\mathbf{k}, t)$, (un $)_{j q}^{*}(\mathbf{k}, t)$ - Fourier-images of functions given on the surface of a deformable body and representing the product of the $\mathrm{j}$ coordinate of the displacement vector on the $\mathrm{q}$ coordinate of the normal to the surface.

Values $P_{j u}^{*}(\mathbf{k}, t)$ and $(u n)_{j q}^{*}(\mathbf{k}, t)$ concerning (49) are unknown, their determination was carried out by using the known solutions of the first and second boundary value problems of the static theory of elasticity when writing the displacement vector ui $(x, t)$ in the form of a volume potential is:

$$
u_{i}(\mathbf{x}, t)=\int_{V_{0}} K_{i j}(\mathbf{x}-\mathbf{y}, t) F_{j}(\mathbf{y}, t) d \mathbf{y}
$$

The displacement vector given by (27), by virtue of the properties of the volume potential, satisfies the system of equations of the boundary value problem (47), taking into account that $\mathrm{x}$ belongs to the surface $\mathrm{S}$.

The integration over the surface of the S-boundary of the body, the application of the Ostrogradskii-Gauss formula, the convolution theorem on the volume, and the implementation of a number of transformations, taking into account the division of the body surface into parts $\mathrm{Su}(\mathrm{t}), \mathrm{S} \delta(\mathrm{t})$ made it possible to get:

$$
\begin{aligned}
& \left\{K_{i m}^{*}(\mathbf{k}, t)-K_{i j}^{* u}(\mathbf{k}, t) i \mathbf{k} q K_{i q m}^{*}(\mathbf{k}, t)+\left[i k_{q} \cdot K_{i n}^{*}(\mathbf{k}, t)+K_{i m}^{*}, q(\mathbf{k}, t)\right] \times\right. \\
& \left.\times(\sigma)_{i m q}^{* \sigma}(\mathbf{k}, t)\right\} \cdot F_{m}^{*}(\mathbf{k}, t)=\left[K_{i j q}^{* \sigma}(\mathbf{k}, t)-K_{i j}^{u}(\mathbf{k}, t)\right] P_{j}^{*}(\mathbf{k}, t)+ \\
& +\left[(\sigma)_{i j q}^{* \sigma}(\mathbf{k}, t)-(\sigma)_{i j q}^{* u}(\mathbf{k}, t)\right](u n)_{j q}^{*}(\mathbf{k}, t)
\end{aligned}
$$

Relation (28) is a system of three equations $(i=1,2,3)$ for defining three unknown functions $F_{m}(\mathbf{k}, t)$ and is integral type of initial boundary problem. Matrix introduction $R_{m i}(\mathbf{x}, t)$,and inverse system matrix allowed to put down the solution of the system as follows:

$$
\begin{aligned}
& F_{m}^{*}(\mathbf{k}, t)=R_{m i}(\mathbf{k}, t)\left\{\left[K_{i j}^{* \sigma}(\mathbf{k}, t)-K_{i j}^{* u}(\mathbf{k}, t)\right] P_{j}^{*}(\mathbf{k}, t)+\right. \\
& \left.+\left[(\sigma)_{i j q}^{* \sigma}(\mathbf{k}, t)-(\sigma)_{i j q}^{* u}(\mathbf{k}, t)\right](u n)_{i q}^{* u}(\mathbf{k}, t)\right\}
\end{aligned}
$$

The relations (27) and (29) allow to write down the solution of the original problem in the form

$$
\begin{aligned}
& u_{i}(\mathbf{x}, t)=\int_{V_{0}} K_{i n}(\mathbf{x}-\mathbf{y}, t) \frac{1}{(2 \pi)^{3}} \int_{R^{3}} e^{-i k \cdot y} \cdot R_{m k}(\mathbf{k}, t)\left\{\left[K_{h j}^{* \sigma}(\mathbf{k}, t)-K_{h j}^{* u}(\mathbf{k}, t)\right] \times\right. \\
& \left.\times P_{j}^{*}(\mathbf{k}, t)+\left[(\sigma)_{h j q}^{* \sigma}(\mathbf{k}, t)-(\sigma)_{h j q}^{*}(\mathbf{k}, t)\right](u n)_{j q}^{* u}(\mathbf{k}, t)\right\} d \mathbf{k} d \mathbf{y}
\end{aligned}
$$

\section{Conclusions}

From the evidence presented in the paper and the method proposed by the authors, it follows:

1. The quadrature (30) satisfies the system of differential equations of the boundary value problem (24).

2. The quadrature (30) satisfies the boundary conditions of the problem (24).

3. The quadrature (30) is a solution of the boundary-value problem (24). 
Thus, the problem of creating an effective method for calculating the deformed state of homogeneous anisotropic bodies of complex shape under static loading with external influences varying in magnitude, direction, and area of application is proposed and considered in this paper.

\section{References}

1. V. Yu. Veshchin, et al, Actual problems in construction and architecture. SamGASA, 69-71 (2002)

2. I. L. Golenkov, V. Yu. Veshchin, et al, Actual problems in construction and architecture. SamGASA, 66-68 (2002)

3. I. S. Kholopov, A. V. Soloviev, et al, Actual problems in construction and architecture. SamGASA, 279-280 (2004)

4. V. A. Kazhaev, et al, Building-info: newsletter 16 (232), 20-31 (2004)

5. I. S. Kholopov, A. V. Soloviev, et al, Actual problems in construction and architecture. SamGASA, 370 (2006)

6. V. Yu. Alpatov, Actual problems in construction and architecture. SamGASA, 474 (2006)

7. A. V. Soloviev, I. S. Kholopov, et al, Metal constructions 15 (1), 39-46 (2009)

8. G. Yu. Ermolenko, Stress-strain state of elastic and viscoelastic finite bodies of arbitrary shape under static and dynamic loading (Samara, SGAU, 2001)

9. B. G. Ivanov, Thesis for a Doctoral Degree in Technical Sciences, (2007)

10. B. G. Ivanov, Izvestiya of Samara Scientific Center of Russian Academy of Sciences, Special. issue Problems of railway transport at the present stage of development, (2006)

11. B. G. Ivanov, Aggregates of the railway life support system with lever-articulated kinematic connections (Samara, Samara State Technical University, 2006)

12. T. B. Dormidontova, V. P. Popov, Scientific Review 4, 19-24 (2014)

13. V. Yu. Alpatov, O. Yu. Veremeenko, et al, MATEC Web Conf. 86, 02015 (2016)

14. S. Petrov, et al, MATEC Web Conf. 106, 04002 (2017)

15. V. Yu. Alpatov, Postgraduate Student of Samara province 2, 22-24 (2001)

16. V. Yu. Alpatov, Effective Building Constructions: a collection of articles of the International Scientific and Technical Conference, 346-352 (2002)

17. V. Yu. Alpatov, Procedia Engineering 91, 177-182 (2014)

18. A. A. Lukin, et al, Procedia Engineering 111, 20-29 (2015)

19. V. Yu. Alpatov, MATEC Web Conf. 86, 02020 (2016)

20. M. I. Balzannikov, I. S. Kholopov, et al, Procedia Engineering 111, 72-81 (2015)

21. M. I. Balzannikov, I. S. Kholopov, et al, MATEC Web Conf. 73, (2016)

22. V. Yu. Alpatov, MATEC Web Conf. 86, 02005 (2016)

23. I. S. Kholopov, M. I. Balzannikov, et al, Procedia Engineering 153, 277-282 (2016)

24. A. A. Lukin, I. S. Kholopov, et al, Procedia Engineering 153, 414-418 (2016)

25. V.Yu. Alpatov, A. A. Sakharov, et al, MATEC Web Conf. 86, 02021 (2016)

26. V. Yu. Alpatov, A. A. Sakharov, et al, MATEC Web Conf. 117, 00007 (2017) 
27. A. M. Mushkat, et al., Traditions and innovations in construction and architecture. Construction, 463-464 (2008)

28. I. S. Kholopov, A. V. Soloviev, et al, Effective constructions, materials and technologies in construction and architecture: a collection of articles of the International Conference, 3-5 (2009)

29. A.V. Soloviev, I. S. Kholopov, et al, Effective designs, materials and technologies in construction and architecture: a collection of articles of the International Conference, 122-127 (2009)

30. I. C. Kholopov, A. B. Soloviev, et al, Collection of reports of the scientific-practical conference dedicated to the 100th anniversary of the birth of Professor E.I. Beleni "Calculation and design of metal structures", 236-241 (2013)

31. I. C. Kholopov, A. B. Soloviev, et al, Science and Education in the 21 st Century: a collection of scientific papers on the materials of the International Scientific and Practical Conference, 145-147 (2013)

32. A. O. Lukin, A. A. Sakharov, et al, Industrial and Civil Engineering 9, 9-14 (2015) 